\title{
State-Specified Protonium Formation in Low-Energy Antiproton-Hydrogen-Atom Collisions
}

\author{
X. M. Tong, ${ }^{1,2, *}$ K. Hino, ${ }^{1,2}$ and N. Toshima ${ }^{1}$ \\ ${ }^{1}$ Institute of Materials Science, Graduate School of Pure and Applied Sciences, University of Tsukuba, \\ 1-1-1 Tennodai, Tsukuba, Ibaraki 305-8573, Japan \\ ${ }^{2}$ Center for Computational Sciences, University of Tsukuba, 1-1-1 Tennodai, Tsukuba, Ibaraki 305-8577, Japan
}

(Received 1 June 2006; published 15 December 2006)

\begin{abstract}
We calculate state-specified protonium-formation cross sections in low-energy antiproton-hydrogenatom collisions by solving the Chew-Goldberger-type integral equation directly instead of integrating the traditional differential scattering equation. Separating the incident wave from the total wave function, we calculate only the scattered outgoing wave propagated by the Green function. The scattering boundary condition is hence automatically satisfied without the tedious procedure of adjusting the wave function at the asymptotic region. The formed protonium atoms tend to be distributed in higher angular momentum $\ell$ and higher principle quantum number $n$ states as the collision energy increases. The present method has the advantage over the traditional ones in the sense that the required memory size and the computational time are much smaller, and accordingly the problem can be solved with higher accuracy.
\end{abstract}

The interaction of the antiproton $(\bar{p})$ with matter is one of the hot topics in recent atomic physics since the discovery of long-lived antiprotonic helium [1,2] and the production of cold antihydrogen atoms [3,4]. In the former event, the antiproton plays a role of a heavy "electron" in the formed metastable atoms and presents interesting features of an exotic Coulomb three-body system. In the latter case, the antihydrogen is expected to provide important information on the symmetry of the antimatter and matter properties and interactions. Ultraslow antiproton beams below $10 \mathrm{eV}$ will be available in the antiproton decelerator facility at CERN in a near future, and collision experiments with atoms and molecules are planned [5]. A theoretical study of antiproton collisions is also desired in harmony with the rapid development of experimental research in order to analyze experimental data or to design the collision facility in which antiprotons can be lost through the annihilation with protons.

The collision of an antiproton with atomic hydrogen is one of the most fundamental atomic processes and serves as a prototype for studying the peculiarity of massive negatively charged particles. Protonium formation occurs efficiently at low energies below the ionization threshold as

$$
\bar{p}+\mathrm{H}(1 s) \rightarrow \bar{p} p+e .
$$

Because of the large mass difference between the antiproton and the electron, protonium atoms are produced preferentially in highly excited states and many formation channels are inevitably coupled. As shown in a recent work of the hyperspherical coordinate method by Esry and Sadeghpour [6], a huge number of the adiabatic potentials had to be taken into account and they could not solve the scattering equations without reducing the antiproton mass artificially. Protonium-formation cross sections were calculated by scaling down the proton and antiproton masses to 20 a.u. Hesse et al. [7] used the diabatization technique to calculate the protonium formation, but they were also obliged to use scaled proton and antiproton masses of 100 a.u. Their formation cross sections are peaked at $n=7$, which is much lower than the estimated value of $n=30$ for the protonium with the real mass, 1836 a.u. There is no definite way to convert the $n$ distribution of the scaled-mass calculations to that of the real system. Cohen [8] calculated the protonium-formation cross section by the classical trajectory Monte Carlo method. The applicability of the classical mechanics at such low energies is not clear, especially for the motion of the electron. Besides, ambiguity occurs in extracting the discrete quantum numbers $n$ and $\ell$ from the classical continuous quantities.

Sakimoto [9] studied the formation process by the timedependent wave-packet method but he did not obtain statespecified formation cross section due to the numerical difficulty. He calculated the formation probability by subtracting the sum of elastic and excitation probabilities from unity. In order to localize the wave packet in a sufficiently small region, one has to superpose a bunch of states belonging to different collision energies, so keeping high accuracy throughout the collision is not easy. Recently, Ovchinnikov and Macek [10] applied the advanced adiabatic approach and presented $n$-dependent formation cross sections. Although their sophisticated method may be accurate qualitatively for the relevant process, it is not easy to estimate the quantitative reliability for the minute information such as the $n$ distribution. To the best of our knowledge, reliable state-specified formation cross sections have not been obtained yet from nonperturbative quantal calculations.

As mentioned above, the traditional coupled-channel method for the time-independent differential Schrödinger equation is not practically applicable to the present process (1) because the required number of channels is as large as a 
few thousand or more. The only way to solve the scattering equation is the direct integration method in which the coordinate space (real or momentum) is segmented by introduction of a mesh and the wave function is represented as discretized quantities on the grid points. For this purpose, we start from the Chew-Goldberger integral representation of the scattering equation (atomic units are used hereafter unless otherwise stated):

$$
\Psi^{+}=\Psi_{0}+\frac{1}{E_{0}-H+i \eta} V_{i n} \Psi_{0} .
$$

$\Psi_{0}$ stands for the initial wave function in which the interaction $V_{\text {in }}$ is absent between the projectile and the target atom. In the denominator of the Green function, $H$ and $E_{0}$ are the Hamiltonian of the whole system and its eigenenergy, respectively, and $\eta$ is a positive infinitesimal quantity. $\Psi^{+}$is the total wave function satisfying the scattering boundary condition of the outgoing wave. For the threebody system, the six dimensions of the coordinates can be reduced to three dimensions in the body-fixed frame after separating the Euler angles representing the direction of the plane containing the three particles. Suppose we try to solve Eq. (2) numerically using $N$ mesh points in each dimension; then the total number of the mesh points is $N^{3}$, the required memory size to store the Green function is $N^{6}$, and the number of the operations to invert the $N^{3} \times N^{3}$ matrix is $N^{9}$. If we use $N=100$, for instance, the number of operations becomes an astronomical quantity, too large even for a modern fast parallel computer.

Introduction of a time-dependent representation gives a clue to overcome this difficulty. Encouraged by the success of the recent studies based on the time-dependent methods $[9,11,12]$, we develop a numerical procedure converting Eq. (2) to

$$
\begin{aligned}
\Psi^{+} & =\Psi_{0}-i \int_{-\infty}^{0} e^{-i\left(E_{0}-H\right) t} V_{\text {in }} e^{\eta t} \Psi_{0} d t, \\
& =\Psi_{0}-i \int_{-\infty}^{0} e^{-i E_{0} t} U(0, t) V_{\text {in }} e^{\eta t} \Psi_{0} d t .
\end{aligned}
$$

Note that the two equations (2) and (3) are mathematically equivalent. At the sacrifice of introducing an additional redundant dimension of time, we can reduce the computational labor considerably. The memory size required for $N^{3}$ mesh points is $N^{4}$, and the number of operations increases only as $N^{4} N_{t}$, where $N_{t}$ is the number of time steps for the numerical time integration.

The formulation is general for arbitrary three-body systems so far. Going back to the specified process (1), we show how to obtain the dynamical information by solving Eq. (3) numerically. In the center of mass frame, we choose the Jacobi coordinates in the space-fixed frame in such a way that $\mathbf{r}$ represents the electron position relative to the proton and $\mathbf{R}$ the antiproton position relative to the center of mass of the hydrogen atom. In this coordinate system, the total Hamiltonian is written as

$$
\begin{aligned}
H & =-\frac{1}{2 \mu_{e}} \nabla_{\mathbf{r}}^{2}-\frac{1}{2 \mu_{p}} \nabla_{\mathbf{R}}^{2}-\frac{1}{r}-\frac{1}{\left|\mathbf{R}+a_{e} \mathbf{r}\right|}+\frac{1}{\left|\mathbf{R}-a_{p} \mathbf{r}\right|}, \\
\mu_{e} & =\frac{m_{e} M_{p}}{\left(M_{p}+m_{e}\right)} \quad \text { and } \quad \mu_{p}=\frac{\left(M_{p}+m_{e}\right) M_{\bar{p}}}{\left(M_{p}+M_{\bar{p}}+m_{e}\right)}
\end{aligned}
$$

where $m_{e}, M_{p}$, and $M_{\bar{p}}$ are the electron, proton, and antiproton masses, respectively. The ratios $a_{e}$ and $a_{p}$ are defined as $a_{e}=m_{e} /\left(M_{p}+m_{e}\right)$ and $a_{p}=M_{p} /\left(M_{p}+\right.$ $\left.m_{e}\right)$. Since the total angular momentum $L$ and its projection $(M)$ on the incident velocity (v) direction and the parity $\pi$ are good quantum numbers, the total wave function for given $L, M$, and $\pi$ can be expressed as

$$
\Psi_{L M}(\mathbf{r}, \mathbf{R}, t)=\sum_{\alpha} F^{\alpha}(r, R, t) \Omega_{L M}^{\alpha},
$$

with

$$
\Omega_{L M}^{\alpha}=\sum_{m_{e}, m}\left\langle l_{e} m_{e} l m \mid L M\right\rangle Y_{l_{e} m_{e}}(\hat{r}) Y_{l m}(\hat{R}),
$$

where $\left(l_{e}, m_{e}\right)$ and $(l, m)$ are the angular momentum quantum numbers corresponding to the coordinates $\mathbf{r}$ and $\mathbf{R}$, respectively, and $\alpha=\left(l_{e}, l\right)$. In the partial wave expansion given above, the total Hamiltonian can be recasted as

$$
\begin{aligned}
\left\langle\Omega_{L M}^{\alpha}|H| \Omega_{L M}^{\alpha^{\prime}}\right\rangle= & \left(-\frac{1}{2 \mu_{p}} \frac{\partial^{2}}{\partial R^{2}}+\frac{l(l+1)}{2 \mu_{p} R^{2}}-\frac{1}{R}\right) \delta_{\alpha, \alpha^{\prime}} \\
& +\left(-\frac{1}{2 \mu_{e}} \frac{\partial^{2}}{\partial r^{2}}+\frac{l_{e}\left(l_{e}+1\right)}{2 \mu_{e} r^{2}}-\frac{1}{r}\right) \delta_{\alpha, \alpha^{\prime}} \\
& +\left\langle\Omega_{L M}^{\alpha}\right| \frac{1}{R}-\frac{1}{\left|\mathbf{R}+a_{e} \mathbf{r}\right|} \\
& +\frac{1}{\left|\mathbf{R}-a_{p} \mathbf{r}\right|}\left|\Omega_{L M}^{\alpha^{\prime}}\right\rangle \\
= & H^{\alpha}(R)+H^{\alpha}(r)+V^{\alpha, \alpha^{\prime}}(r, R) .
\end{aligned}
$$

The time propagation in Eq. (3) can be carried out by the split-operator method [13] as

$$
\begin{aligned}
U^{\alpha^{\prime}, \alpha}(t+\Delta, t)= & e^{-i H^{\alpha^{\prime}}(R) \Delta t / 2} e^{-i H^{\alpha^{\prime}}(r) \Delta t / 2} e^{-i V^{\alpha^{\prime}, \alpha}(r, R) \Delta t} \\
& \times e^{-i H^{\alpha}(r) \Delta t / 2} e^{-i H^{\alpha}(R) \Delta t / 2}+O\left(\Delta t^{3}\right) .
\end{aligned}
$$

We split the time propagation into five successive steps: propagate the wave function under (i) $H^{\alpha}(R)$ for a half time step, (ii) $H^{\alpha}(r)$ for a half time step, (iii) $V^{\alpha^{\prime}, \alpha}(r, R)$ for a full time step, (iv) $H^{\alpha^{\prime}}(r)$ for a half time step, and finally (v) $H^{\alpha^{\prime}}(R)$ for a half time step. The numerical advantage of the split-operator method is that we propagate only one variable by fixing the others in each step. We use the generalized pseudospectral method [13,14], which enables us to depict the bound and continuum wave functions with a modest number of the grid, in order to discretize the coordinate space of $(r, R)$. We add an energy-dependent optical potential [15] at the space boundary to absorb the outgoing wave and eliminate the unphysical reflection. The 
initial wave function is expressed as

$$
\Psi_{0}(\mathbf{r}, \mathbf{R})=\psi_{1 s}(\mathbf{r}) e^{i \mathbf{k}_{0} \cdot \mathbf{R}}=\sum_{L M} \Psi_{0}^{L M},
$$

with $\mathbf{k}_{0}$ the incident momentum of the antiproton in the center of mass coordinates. Since $M_{p} \gg m_{e}$, we can safely set $a_{e}=0$ and $a_{p}=1$ in the potential terms of Eq. (4). We confirmed numerically that the resulting error is negligibly small. We also found that the replacement of $\exp (\eta t)$ by $\exp \left(-t^{2} / \tau^{2}\right)$ in Eq. (3) gives the same results with less propagation time. Therefore, in the calculations that we carry out, we replace $\exp (\eta t)$ by $\exp \left(-t^{2} / \tau^{2}\right)$ in order to accelerate the numerical evolution,

$$
\begin{gathered}
\Psi_{L M}^{+}=-i \int_{-\infty}^{0} e^{-i\left(E_{0}-H\right) t} e^{-t^{2} / \tau^{2}} V_{\text {in }} \Psi_{0}^{L M} d t+\Psi_{0}^{L M}, \\
V_{\text {in }}=\frac{1}{|\mathbf{R}-\mathbf{r}|}-\frac{1}{R}
\end{gathered}
$$

In the integral of Eq. (9), $V_{\text {in }} \Psi_{0}$ produces a wave packet emerging from the origin and propagating to the outside in contrast with the time-dependent wave-packet method.

The state-specified formation cross section can be expressed as

$$
\sigma_{L M}^{n l}=\frac{4 \mu_{p} \mu_{e} k_{n l}}{k_{0}} \sum_{\alpha}\left|T_{n l}^{\alpha}\right|^{2},
$$

and the $T$-matrix element is given by

$$
T_{n l}^{\alpha}=\left\langle j_{l_{e}}\left(k_{n l} r\right) \psi_{n l}(R) \Omega_{L M}^{\alpha}\left|\frac{1}{|\mathbf{R}-\mathbf{r}|}-\frac{1}{r}\right| \Psi_{L M}^{+}\right\rangle,
$$

with $k_{n l}$ the outgoing electron momentum associated with the formation of protonium in the $n l$ state. Here we have replaced $\left|\mathbf{R}+a_{e} \mathbf{r}\right|$ by $R$ in $\psi_{n l}$ since the geometrical distribution of the protonium wave function differs little by this replacement. In the present calculations, we employ about 400 grid points in $R, 200$ grid points in $r$, and 6 channels in $\alpha$. Tens of thousand to hundreds of thousand time steps have been used in the calculation. We carefully checked the convergence of the integrals by varying the number of grid points in each coordinate, the number of coupled channels, and the propagation time. We also confirmed that the results are independent of the choice of the parameter $\tau$ if it is sufficiently large. More details of the numerical procedure and the extensive convergence test will be presented elsewhere [16].

Figure 1 shows the total formation cross section $\sigma_{T}=$ $\sum_{L M} \sum_{n l} \sigma_{L M}^{n l}$. Our results are in reasonable agreement with the recent wave-packet calculations [9]. Since our total cross sections are obtained as the sum of the individual formation cross sections differently from the wavepacket calculations, the agreement demonstrates the reliability and effectiveness of our method.

Figure 2 shows the total angular momentum dependent protonium-formation cross sections $\left(\sigma_{L}=\sum_{M} \sum_{n \ell} \sigma_{L M}^{n \ell}\right)$.

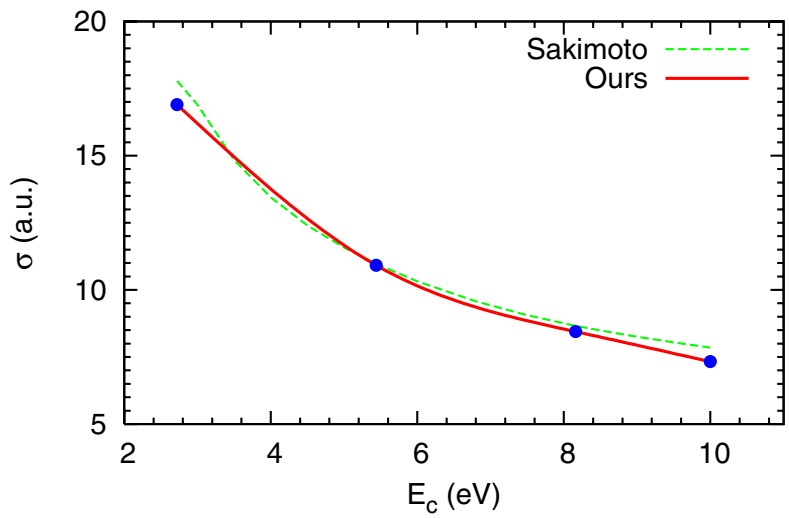

FIG. 1 (color online). Total protonium-formation cross sections for the incident energy below $n=2$ excitation threshold. The results (dashed curve) from Ref. [9] are also presented for comparison.

At the lowest energy $(2.72 \mathrm{eV})$ the formation cross sections increase monotonically from $L=0$ to $L=30$ and then drop sharply to zero. As the collision energy increases, the peak position shifts to higher $L$ and the distribution becomes broader. At the highest energy $(10.0 \mathrm{eV})$ the cross sections take the maximum value at $L=38$ and then decrease slowly as the angular momentum $L$ increases further. The present results of the $L$ dependence also agree well with those of Sakimoto [9]. The formation cross sections increase nearly in proportion to the statistical weight $2 L+1$ below the peak points indicating that the formation probabilities are almost constant in this region. Interpretation and discussions on this behavior are given in the paper of Sakimoto [9] in the comparison with his semiclassical calculations.

Figure 3 shows a two-dimensional plot of the statespecified ( $n$ - and $l$-dependent) cross sections $\sigma_{n \ell}=$ $\sum_{L M} \sigma_{L M}^{n \ell}$ at $10 \mathrm{eV}$. We see that the protonium atoms are mainly formed in the states with the angular momentum $\ell \sim 38$ and the principal quantum number $n \sim 50$. The

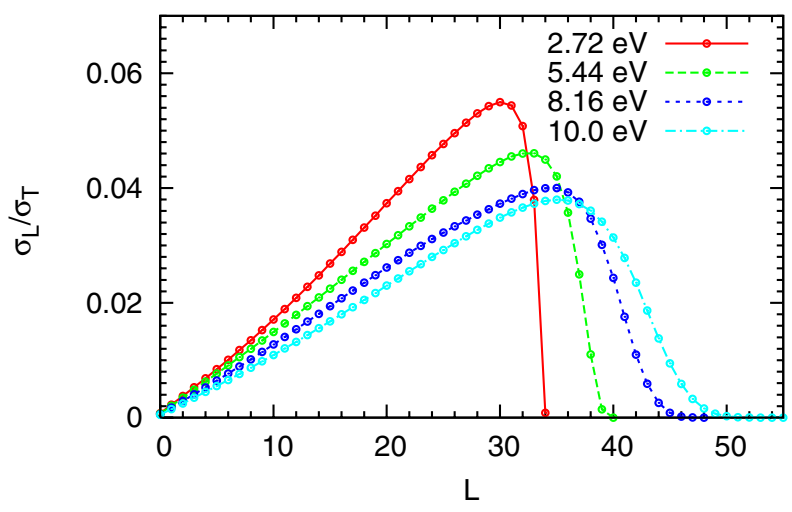

FIG. 2 (color online). Total angular momentum dependent protonium-formation cross sections normalized to the total formation cross section at the incident energies of 2.72, 5.44, 8.16, and $10 \mathrm{eV}$, respectively. 


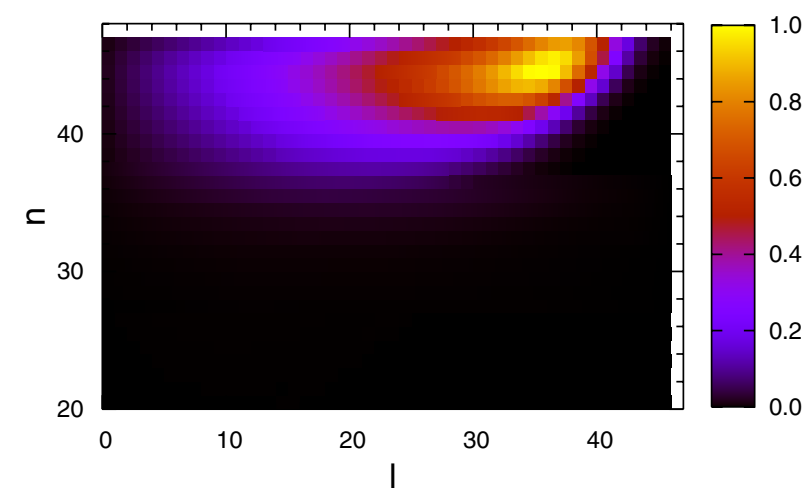

FIG. 3 (color online). $n$ - and $\ell$-dependent protoniumformation cross sections at $10 \mathrm{eV}$ incident energy.

probability of formation in the lower $n$ decreases quickly. Although the two-dimensional image can give a global feature well, minute information is not easy to be presented from it. We give $\ell$-dependent cross sections $\left(\sigma_{\ell}=\sum_{n} \sigma_{n \ell}\right)$ and $n$-dependent cross sections $\left(\sigma_{n}=\sum_{\ell} \sigma_{n \ell}\right)$ in Figs. 4(a) and 4(b), respectively. It is interesting to see that the $\ell$ dependence is similar to the $L$ dependence given in Fig. 2. The similarity indicates that the heavy particles mainly carry the angular momentum, and its exchange between
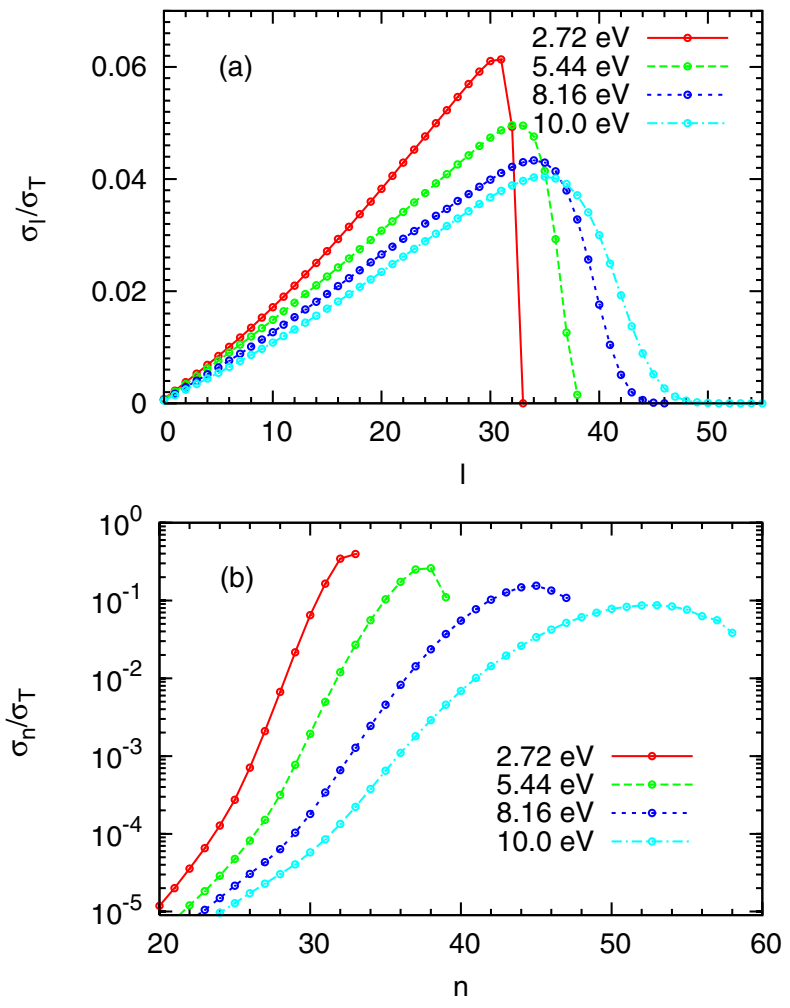

FIG. 4 (color online). (a) $\ell$ - and (b) $n$-dependent protoniumformation cross sections at the incident energies 2.72, 5.44, 8.16, and $10 \mathrm{eV}$, respectively. the electron and the antiproton is small. The $n$ distribution of the formation cross sections shows large energy dependence at variance with the simple estimate that, based on the energy matching consideration between the initial and final bound states, the protonium is mainly formed in the states with $n \sim \sqrt{\mu} \sim 30$, where $\mu$ is the reduced mass of the protonium. This prediction holds only for the lowest incident energy. As the incident energy increases, the $n$ distribution becomes broader and the peak position moves to higher $n$.

To summarize, we have developed a time-dependent scattering theory which is very efficient in low-energy collisions where many channels are coupled and traditional coupled-channel methods are not applicable. In this formulation, only the outgoing part of the scattering equation is calculated by direct numerical integration without basis expansion. In contrast to the wave-packet theory, only monochromatic collision energy is included so that high precision calculations are possible. Based on this theory, we studied the state-specified protonium-formation in antiproton-hydrogen-atom collisions. We find that protonium atoms are formed in states with higher $n$ than the simple estimate $n=30$. The present method is general and it can be applied to many other collision processes. The usefulness and effectiveness of the method will be explored and demonstrated in future studies.

This research is supported by Grant-in-Aids for Scientific Research (B) from the Japan Society for the Promotion of Science.

*Electronic address: tong@ims.tsukuba.ac.jp

[1] T. Yamazaki, N. Morita, R. S. Hayano, E. Widmann, and J. Eade, Phys. Rep. 366, 183 (2002).

[2] M. Iwasaki et al., Phys. Rev. Lett. 67, 1246 (1991).

[3] M. Amoretti et al., Nature (London) 419, 456 (2002).

[4] G. Gabrielse et al., Phys. Rev. Lett. 89, 213401 (2002).

[5] N. Kuroda et al., Phys. Rev. Lett. 94, 023401 (2005).

[6] B. D. Esry and H. R. Sadeghpour, Phys. Rev. A 67, 012704 (2003).

[7] M. Hesse, A.T. Le, and C.D. Lin, Phys. Rev. A 69, 052712 (2004).

[8] J. S. Cohen, Phys. Rev. A 36, 2024 (1987).

[9] K. Sakimoto, Phys. Rev. A 65, 012706 (2002).

[10] S. Y. Ovchinnikov and J.H. Macek, Phys. Rev. A 71, 052717 (2005).

[11] D. R. Schultz, M. R. Staryer, and J. C. Wells, Phys. Rev. Lett. 82, 3976 (1999).

[12] X. M. Tong, T. Watanabe, D. Kato, and S. Ohtani, Phys. Rev. A 64, 022711 (2001).

[13] X. M. Tong and S. I. Chu, Chem. Phys. 217, 119 (1997).

[14] X. M. Tong and S.I. Chu, Phys. Rev. A 61, 031401(R) (2000).

[15] J. Perie and G. Jolicard, J. Phys. B 26, 4491 (1993).

[16] X. M. Tong, K. Hino, and N. Toshima (to be published). 